\title{
Radiological and clinical characteristics of 22 suspected COVID-19 patients with multiple negative RT-PCR testing
}

\section{Wenjun Wu}

Huazhong University of Science and Technology

\section{Heshui Shi}

Huazhong University of Science and Technology

\section{Bo Liang}

Huazhong University of Science and Technology

\section{Feihong Wu}

Huazhong University of Science and Technology

\section{Dongqiao Xiang}

Huazhong University of Science and Technology

\section{Ziji Qin}

Huazhong University of Science and Technology

Hongyan Zhou

Huazhong University of Science and Technology

Chuansheng Zheng ( $\nabla$ hqzcsxh@sina.com)

Huazhong University of Science and Technology

Lixia Wang ( $\sim$ lisa2003627_1@163.com )

Huazhong University of Science and Technology

\section{Research Article}

Keywords: Coronavirus infections, $\mathrm{x}$-ray computed Tomography, viral Pneumonia, Thorax, Lung diseases

Posted Date: May 5th, 2020

DOI: https://doi.org/10.21203/rs.3.rs-27135/v1

License: (c) (i) This work is licensed under a Creative Commons Attribution 4.0 International License.

Read Full License 


\section{Abstract}

This study aims to investigate radiological and clinical characteristics of suspected COVID-19 patients with negative RT-PCR testing. 22 suspected COVID-19 patients with 2-5 negative RT-PCR testing were enrolled. The most common symptoms at onset included fever (18[81.8\%] patients), fatigue (16[72.7\%]) and cough (12[54.5\%]). 20(90.9\%) patients were with normal or decreased Leukocyte count, $17(77.3 \%)$ with decreased Lymphocyte count, 16 (72.7\%) and 18 (81.8\%) with increased level of C-reactive protein and D-dimers, respectively. Ground-glass opacity (GGO) (22[100]), reticular/interlobular septal thickening (11 [50\%]) and consolidation (14 [63.6\%)) with predominantly bilateral and peripheral distribution were typical findings at initial CT scan. The CT score of right lower lobe was significantly higher than right upper lobe ( $P=0.042)$. The total CT scores of group 2, 3, 4 (2nd week, 3rd week, 4th week and after) were significantly higher than group 1 (1st week) (P囚0.01,for all). After rapid progression at first week, the most extensive pulmonary involvement emerged at second week after symptom onset, then the involvement gradually decreased. Given the clinical symptoms, laboratory results and typical radiological manifestations, even suspected COVID-19 patients with multiple negative RT-PCR testing deserve great concern for active management.

\section{Highlights}

- Suspected COVID-19 patients with negative RT-PCR testing present typical clinical symptom, laboratory finding and chest CT

- Chest CT manifestations and temporal changes of suspected COVID-19 are characteristic and similar to confirmed COVID-19.

- Clinicians and radiologists should be alerted when encountering suspected COVID- 19 patient with negative RT-PCR

\section{Introduction}

Since December 2019, an outbreak of a novel coronavirus disease (COVID-19) began in Wuhan, Hubei province, China and led to a major concern of the potential for a pandemic because of the rapidly increased number of confirmed cases outside of China. $(1,2)$ The widespread human-to-human transmission of COVID-19 resulted in 113702 confirmed cases in 109 countries/territories/ areas with 4012 deaths as of March 11, 2020. (2-4) Although the exact origin of remained unknown, most early reported cases had an exposure history to the city's Huanan seafood wholesale market where wild animals were sold. $(1,5)$ The pathogen of COVID-19 was identified as a novel enveloped RNA betacoronavirus named severe acute respiratory syndrome coronavirus 2 (SARS-CoV-2).(6) Despite of the phylogenic heterogeneity of SARS- CoV-2 from the known human-infected coronavirus such as severe acute respiratory syndrome coronavirus (SARS-CoV) and Middle East respiratory syndrome coronavirus (MERS-CoV),(7) infection of SARS-CoV-2 could also induce severe respiratory infection symptoms, even 
acute respiratory distress syndrome (ARDS), acute respiratory failure, and other serious complications.(1, 5)

Although the mortality of COVID-19 is lower than SARS and MERS, its stronger human-to-human transmission possibly overwhelm the healthcare systems. (6) Besides, the current lack of curative vaccine makes early diagnosis and efficient control of transmission route the most available way in the battle with COVID-19. As multiple studies have focused on the epidemiologic, laboratory and clinical features of COVID-19 in order to understand the evolution of COVID-19 and control disease spread, some early radiologists have also profiled the imaging patterns on chest computed tomography (CT). (8-12) In confirmed COVID-19 cases, the characteristics and distribution of pulmonary lesions were specific to some extent. For instance, ground glass opacity (GGO) and consolidation were the most common findings on chest $\mathrm{CT}$, and the distribution of lung lesions were predominantly peripheral subpleural and bilateral pattern.(8-10) As the disease progressed, the involvement of lung extended and the frequency of complex signs such as "crazy-paving" pattern and "reverse-halo" sign increased.(13) In recovered cases, pulmonary abnormalities on chest CT showed the most extensive lesions approximately 10 days after initial onset of symptoms and gradually resolved. $(11,13,14)$ Therefore, chest CT played a vital role in the diagnosis and evaluation of COVID-19.

At present, the confirmation of COVID-19 depends on positive detection of virus nucleic acid with realtime reverse transcriptase polymerase chain reaction (RT-PCR) of respiratory secretions from throat swabs or lower respiratory tract. $(6,7)$ However, clinical practice and published literatures have demonstrated that some patients with negative RT-PCR result for COVID-19 had typical clinical symptoms and chest CT findings of viral pneumonia, and the sensitivity of RT-PCR in detecting COVID-19 seemed lower than that of chest CT. $(15,16)$ In this study, serial chest CT images of 22 suspected COVID-19 patients with twice or more times negative RT-PCR results were characterized and evaluated for the radiological and clinical features.

\section{Results}

There were 13 male and 9 female patients with mean age of 50 years (ranging from 30-79 with standard deviation of 14 years) in this study. All patients had resident and/or exposure history including 6 (27.3\%) medical staffs in the hospital. The most common clinical symptoms at onset were fever (18[81.8\%] patients), fatigue (16[72.7\%]) and cough (12[54.5\%]). 20 (90.9\%) patients had normal or decreased Leukocyte count, 17 (77.3\%) had decreased Lymphocyte count, $16(72.7 \%)$ and 18 (81.8\%) had increased level of C-reactive protein and D-dimers, respectively. All patients demonstrated consecutively at least twice (sampling interval of at least 24 hours) negative detection of virus nucleic acid for COVID-19 via RTPCR. 20 (90.9\%) patients underwent at least twice CT scans during the study period, except for 2 (9.1\%) deceased cases with only initial CT scan. A total of 70 chest CT scans was performed. Each patient underwent an average of $3 \pm 1$ CT scans (range from 1 to 6 times) with a mean interval of $7 \pm 4$ days (range from 3 to 20 days). (Table 1 ) 
Of the 22 patients, all (100\%) had ground glass opacity (GGO), 11 (50\%) had reticular and/or interlobular septal thickening including "crazy-paving" pattern, and 14 (63.6\%) had consolidation at initial CT scan. 1 (4.5\%) patients had "reverse halo" sign during follow-up CT scan. (Supplementary Material 1) The distribution of these lesions demonstrated obvious peripheral tendency with $15(68,2 \%)$ predominantly subpleural, 1 (4.5\%) random and $6(27.3 \%)$ diffuse pattern. (Figure 1) The majority of patients had bilateral lesions (16[72.7\%]) and multiple involved lobes (17[77.3\%]). (Table 2) No patient had mediastinal lymphadenopathy and $2(9.1 \%)$ patients had pleural effusion at initial or follow-up CT scan. Both lower lobes had relatively higher $\mathrm{CT}$ scores than those of middle and upper lobes. The CT score of right lower lobe was significantly higher than right upper lobe $(P=0.042)$. (Table3)

Based on the grouping method, group 1, 2, 3 and 4 included 18, 11, 18 and 23 CT scans, respectively. The mean CT score of all groups was 8.14 \pm 4.58 , with significantly higher CT score of group 2, 3, 4 than group 1 (p囚0.01). Group 2 had the highest CT score of $10.00 \pm 4.58$. (Table 4, Figure 2) According to the evolution of the chest CT features, $11(50 \%)$ patients were determined as resolution and $9(40.9 \%)$ were partial resolution, except for $2(9.1 \%)$ deceased cases with only initial CT scan. Among patients with resolution, the typical process of chest CT manifestation was initial progression to peak level followed by reduced extent and decreased density of pulmonary lesions (Figure 3) or improvement from the most extensive lung involvement. Among patients with partial resolution, the latest chest CT always showed remaining reticular/interlobular septal thickening and/or consolidation. (Figure 4) The initial CT scans of the deceased cases showed relatively large involvement of the lung. (Supplementary Material 2)

\section{Discussion}

As the novel coronavirus disease (COVID-19) rapidly spreads throughout the world, suspected COVID-19 cases with consecutive negative RT-PCR results deserve high attention in the disease control and patient management.(4) According to the current diagnostic criterion, viral nucleic acid test via RT-PCR is the "gold standard" for the confirmation of COVID-19. However, given the limited number and time consuming process of RT-PCR kits in some medical centers and the possibility of false negative results via RT-PCR, clinicians and radiologists probably encounter and interpret suspected COVID-19 cases without timely viral nucleic acid test or with negative result. $(7,15,16)$ Actually, COVID-19 is contagious even in incubation period regardless of the RT-PCR result.(3,6) To avoid over-dependence on RT-PCR results, clinical and imaging analysis of suspected COVID-19 cases is particularly important. Chest CT is supposed to be a more sensitive method in detecting COVID-19 than RT-PCR.(14-16) In this study, we reviewed the clinical and chest CT characteristics of a cluster of patients with SARS-CoV-2 exposure history and fever and/or respiratory symptoms but without positive RT-PCR results for COVID-19. The typical chest CT manifestation of suspected COVID-19 cases may help medical institutions to isolate and treat patients at early stage.

Without positive RT-PCR results, the enrolled suspected COVID-19 cases still showed some according laboratory features including normal or reduced counts of lymphocytes at early stage, elevated C-reactive protein and D-dimers in the majority of patients. Together with the exposure history and clinical 
symptoms, suspected COVID-19 cases were determined. In practice, chest CT was the routine method to detect the pulmonary abnormality of suspected cases. In this study, all of the patients presented ground glass opacity (GGO) and over half of the patients had consolidation and reticular/ interlobular septal thickening at the initial scan. This result was in accordance to earlier investigations with confirmed COVID-19. GGO, consolidation and interstitial abnormality were the most common findings of either confirmed or suspected COVID-19 patients. $(9,10,17-19)$ As the time interval between initial CT scan and symptom onset were various, the pattern of CT manifestation were different as well. The 3 kinds of lesions were often mixed and tended to present simultaneously, especially in patients with extensive involvement. In patients with just symptom onset, focal GGO was the common findings on chest CT.(1114) Additionally, pulmonary involvement was always characterized by a tendency of bilateral multiple lobes involvement and a predominant distribution in peripheral and lower parts of the lung. $(10,17)$ Our results demonstrated that the CT score of right lower lobe was significantly higher than right upper lobe, indicating more extensive involvement of lower lobe. Meanwhile, some other radiologic signs such as mediastinal lymphadenopathy were not present in this series of patients and pleural effusion was found in only one patient at initial CT scan. Based on the typical chest CT findings, clinicians and radiologists would be more confident to distinguish a suspected COVID-19 from other pulmonary infection.

Despite of lack of supportive RT-PCR results, serial CT imaging of suspected COVID-19 patients could reveal the evolution of the disease and help to monitor disease changes. A total of $70 \mathrm{CT}$ scans was evaluated using a CT score system for the extent of the pulmonary involvement in suspected COVID-19 patients during the follow-up. According to the changes of extent and pattern of pulmonary lesions, most patients showed an improvement from the peak level of the disease, except for 2 deceased cases. The extent of disease on CT scans increased markedly from the first week to the second week after symptom onset, then decreased gradually during the third week and after. The time course of suspected COVID-19 on chest CT was similar to previous investigations on confirmed cases. The peak level of the disease emerged at the second week after symptom onset. $(11,14)$ In this cluster of CT scans, the general CT scores after the second showed no marked decrease because some patients did not fully improve during follow-up. In the progressing stage, besides of the enlarged pulmonary lesions, the pattern of lung lesions became more complex including more extensive consolidation and reticular/interlobular septal thickening, and other signs such as reversed halo sign emerged. The findings were largely similar to earlier radiologic investigative effort on confirmed COVID-19 patients. $(9,13)$ In the improving stage, some patients showed almost complete disappearance of GGO, consolidation and interstitial abnormality, while others showed incomplete absorption of these lesions possibly due to the insufficient observation period. In some cases, the pulmonary lesions on follow-up CT scans could presented as shifting from one location to another or "wane and wax". This might be due to the treatment during progressing stage. The evolution of extent and pattern of lung lesions on chest CT had some features and could be used to evaluate the condition of suspected COVID-19 patients.

We acknowledged several limitations in this study. Firstly, the small sample size could not represent all conditions of suspected COVID-19 and larger sampled studies would be needed to understand the natural history of the disease. Secondly, not all the radiological outcomes of each individual were observed in 
this study because of limited period, further studies on prognosis would be more helpful for patient management. Thirdly, the possible medical intervention before in-patient of some patients might affect the interpretation of CT images.

In conclusion, our investigative effort demonstrated that the GGO, consolidation and interstitial abnormality with predominantly bilateral and peripheral distribution was the most common pattern for suspected COVID-19 patients with negative RT- PCR results. Most cases showed a recovering process from suspected COVID-19 on chest CT, despite of 2 deceased cases. The CT score system showed most extensive pulmonary involvement at second week after symptom onset. In the progressing stage, increased extent of mixed pulmonary lesions was found on chest CT. In the improving stage, gradual absorption of pulmonary lesions was the common finding. In some cases, the pulmonary lesions on follow-up CT scans could presented as shifting from one location to another or "wane and wax", which might be due to the treatment during progressing stage. Considering the exposure history, clinical symptoms and laboratory results, for patients with typical CT findings of viral pneumonia, even if the viral nucleic acid test via RT-PCR is negative, clinicians and radiologists should also be alerted and take appropriate preventive measures.

\section{Methods And Materials}

\section{Patients}

This study was approved by the institutional review board of Union Hospital, Tongji Medical College, Huazhong University of Science and Technology, Wuhan. The requirement for informed patient consent was waived by the ethics committee for this retrospective study. This study was performed in accordance with the Strengthening the Reporting of Observational Studies in Epidemiology (STROBE) methodology. The inclusion criteria of this study were based on the preliminary diagnosis and treatment protocols from the National Health Commission of the People's Republic of China,(20) including 1. travel/residence history in regions with reported COVID-19 cases or exposure history to infected/symptomatic patients within 14 days before the onset of illness; 2 . clinical manifestations including fever and/or respiratory symptoms, normal or decreased count of leucocytes and decreased count of lymphocytes at early stage of the disease, radiological characteristics of viral pneumonia (any 2 of the 3 manifestations); and 4. realtime fluorescence polymerase chain reaction (RT-PCR) revealed at least twice negative detection of SARSCoV-2 nucleic acid. Throat swab specimens were collected for real-time RTPCR analysis for all cases. A convenience sampling approach was used to enroll the patients. The clinical data including gender, age, initial symptoms and laboratory results were recorded and analyzed and the chest CT images of these patients were reviewed from 15 January 2020 to 27 February 2020.

\section{Image acquisition and evaluation}

All patients underwent chest CT using two commercial multi-detector CT scanners (Philips Ingenuity Core128, Philips Medical Systems, Best, the Netherlands; SOMATOM Definition AS, Siemens Healthineers, Germany). All of the initial CT scans were performed at $1.25 \mathrm{~mm}$ or $1.5 \mathrm{~mm}$ slice thickness. Some follow- 
up CT scans were performed at $5 \mathrm{~mm}$ slice thickness. All CT images were reviewed by two radiologists ( $\mathrm{L}$. W., W.W.) using the institutional digital database system (Vue PACS, Carestream Health, Canada). Image evaluation was performed independently and final decisions reached by consensus are reported.

For each patient, the initial and follow-up chest CT were evaluated for the presence of the major radiological signs including ground glass opacity (GGO), consolidation, and reticular/interlobular septal thickening. The numbers and location of involved lung lobes were recorded. The distribution of the lesions was described as predominantly subpleural (involving mainly the peripheral one-third of the lung), random (without predilection for subpleural or central regions), or diffuse (continuous involvement without respect to lung segments). Each of the five lobes was assessed for the degree of involvement on a 6 -score scale: 0 , no involvement; $1,<5 \%$ involvement; $2,25 \%$ involvement; $3,26 \%-49 \%$ involvement; 4 , $50 \%-75 \%$ involvement; $5,>75 \%$ involvement. An overall lung "total CT score" was reached by summing the five lobe scores (range from 0 to 25). Based on the time interval between the onset of symptoms and the CT scans, we designated four groups of initial and follow-up CT scans in our study: group 1 (CT scans performed $\leq 7$ days after symptom onset); group 2 (CT scans performed $>8$ to 14 days after symptom onset); group 3 (CT scans performed $>14$ to 21 days after symptom onset); and group 4 (CT scans performed $>21$ days after symptom onset).

\section{Statistical Analysis}

Statistical analyses were performed using IBM SPSS Statistics Software (version 26; IBM, New York, USA). Quantitative data were presented as mean \pm standard deviation (range) and categorical data were presented as frequency (percentage). The CT score of different lobes were compared using paired $t$ test. The total CT score of different groups was described using the box plots. The total CT score across different groups were compared using independent $t$ test. A p-value of $<0.05$ was defined as statistical significance.

\section{Abbreviations}

COVID-19 2019 Coronavirus Disease

RT-PCR reverse transcriptase polymerase chain reaction

CT computed tomography

SARS-CoV-2 severe acute respiratory syndrome coronavirus 2

SARS-CoV severe acute respiratory syndrome coronavirus

MERS-CoV Middle East respiratory syndrome coronavirus

ARDS acute respiratory distress syndrome

GGO ground glass opacity 


\section{Declarations}

This study was funded by Scientific Research Foundation of Wuhan Union Hospital [grant number 02.03.2018-224].

There are no conflicts of interest to declare.

\section{References}

1. Huang C, Wang Y, Li X, Ren L, Zhao J, Hu Y, Zhang L, Fan G, Xu J, Gu X, Cheng Z, Yu T, Xia J, Wei Y, Wu W, Xie X, Yin W, Li H, Liu M, Xiao Y, Gao H, Guo L, Xie J, Wang G, Jiang R, Gao Z, Jin Q, Wang J, Cao B. Clinical features of patients infected with 2019 novel coronavirus in Wuhan, China. The Lancet 2020;395(10223):497-506. doi: 10.1016/s0140- 6736(20)30183-5

2. Li Q, Guan X, Wu P, Wang X, Zhou L, Tong Y, Ren R, Leung KSM, Lau EHY, Wong JY, Xing X, Xiang N, Wu Y, Li C, Chen Q, Li D, Liu T, Zhao J, Li M, Tu W, Chen C, Jin L, Yang R, Wang Q, Zhou S, Wang R, Liu H, Luo Y, Liu Y, Shao G, Li H, Tao Z, Yang Y, Deng Z, Liu B, Ma Z, Zhang Y, Shi G, Lam TTY, Wu JTK, Gao GF, Cowling BJ, Yang B, Leung GM, Feng Z. Early Transmission Dynamics in Wuhan, China, of Novel Coronavirus-Infected Pneumonia. N Engl J Med 2020. doi: 1056/NEJMoa2001316

3. Chan JF-W, Yuan S, Kok K-H, To KK-W, Chu H, Yang J, Xing F, Liu J, Yip CC-Y, Poon RW-S, Tsoi H-W, Lo SK-F, Chan K-H, Poon VK-M, Chan W-M, Ip JD, Cai J-P, Cheng VC-C, Chen H, Hui CK-M, Yuen K-Y. A familial cluster of pneumonia associated with the 2019 novel coronavirus indicating person-toperson transmission: a study of a family cluster. The Lancet 2020;395(10223):514-523. doi: 10.1016/s0140-6736(20)30154-9

4. Organization WH. Coronavirus disease 2019 (COVID-19) Situation Report - 502020.

5. Chen N, Zhou M, Dong X, Qu J, Gong F, Han Y, Qiu Y, Wang J, Liu Y, Wei Y, Xia Ja, Yu T, Zhang X, Zhang L. Epidemiological and clinical characteristics of 99 cases of 2019 novel coronavirus pneumonia in Wuhan, China: a descriptive The Lancet 2020;395(10223):507-513. doi: 10.1016/s0140-6736(20)30211-7

6. Guan W-j, Ni Z-y, Hu Y, Liang W-h, Ou C-q, He J-x, Liu L, Shan H, Lei C-I, Hui DSC, Du B, Li L-j, Zeng G, Yuen K-Y, Chen R-C, Tang C-I, Wang T, Chen P-y, Xiang J, Li S-y, Wang J-I, Liang Z-j, Peng Y-x, Wei L, Liu Y, Hu Y-h, Peng P, Wang J-m, Liu J-y, Chen Z, Li G, Zheng Z-j, Qiu S-q, Luo J, Ye C-j, Zhu S-y, Zhong $\mathrm{N}-\mathrm{s}$. Clinical Characteristics of Coronavirus Disease 2019 in China. New England Journal of Medicine 2020. doi: 1056/NEJMoa2002032

7. Wang D, Hu B, Hu C, Zhu F, Liu X, Zhang J, Wang B, Xiang H, Cheng Z, Xiong Y, Zhao Y, Li Y, Wang X, Peng Z. Clinical Characteristics of 138 Hospitalized Patients With 2019 Novel Coronavirus-Infected Pneumonia in Wuhan, JAMA 2020. doi: 10.1001/jama.2020.1585

8. Pan Y, Guan H. Imaging changes in patients with 2019-nCov. European radiology 2020. doi: 1007/s00330-020-06713-z 
9. Pan Y, Guan H, Zhou S, Wang Y, Li Q, Zhu T, Hu Q, Xia L. Initial CT findings and temporal changes in patients with the novel coronavirus pneumonia (2019-nCoV): a study of 63 patients in Wuhan, China. European radiology 2020. doi: 1007/s00330-020-06731-x

10. Song F, Shi N, Shan F, Zhang Z, Shen J, Lu H, Ling Y, Jiang Y, Shi Emerging Coronavirus 2019-nCoV Pneumonia. Radiology 2020;2020 Feb 6[Online ahead of print].

11. Pan F, Ye T, Sun P, Gui S, Liang B, Li L, Zheng D, Wang J, Hesketh RL, Yang L, Zheng C. Time Course of Lung Changes On Chest CT During Recovery From 2019 Novel Coronavirus (COVID-19) Pneumonia. Radiology; 2020 Feb 13[Online ahead of print].

12. Shi H, Han X, Zheng C. Evolution of CT Manifestations in a Patient Recovered from 2019 Novel Coronavirus (2019-nCoV) Pneumonia in Wuhan, China. Radiology 2020:200269. doi:

10.1148/radiol.2020200269

13. Adam Bernheim, Xueyan Mei, Mingqian Huang, Yang Yang, Zahi A Fayad, Ning Zhang, Kaiyue Diao, Bin Lin, Xiqi Zhu, Kunwei Li, Shaolin Li, Hong Shan, Adam Jacobi, Chung M. Chest CT Findings in Coronavirus Disease-19 (COVID-19) Relationship to Duration of Infection. Radiology;2020 Feb 20[Online ahead of print].

14. Shi H, Han X, Jiang N, Cao Y, Alwalid O, Gu J, Fan Y, Zheng C. Radiological findings from 81 patients with COVID-19 pneumonia in Wuhan, China: a descriptive The Lancet Infectious Diseases 2020. doi: 10.1016/s1473-3099(20)30086-4

15. Fang Y, Zhang H, Xie J, Lin M, Ying L, Pang P, Ji Sensitivity of Chest CT for COVID- 19: Comparison to RT-PCR. Radiology;2020 Feb 19[Online ahead of print].

16. Ai T, Yang Z, Hou H, Zhan C, Chen C, Lv W, Tao Q, Sun Z, Xia L. Correlation of Chest CT and RT-PCR Testing in Coronavirus Disease 2019 (COVID-19) in China A Report of 1014 Cases. Radiology;2020 Feb 26[Online ahead of print].

17. Kanne Chest CT Findings in 2019 Novel Coronavirus (2019-nCoV) Infections from Wuhan, China Key Points for the Radiologist. Radiology;2020 Feb 4[Online ahead of print].

18. Xu Y-H, Dong J-H, An W-m, Lv X-Y, Yin X-P, Zhang J-Z, Dong L, Ma X, Zhang H-J, Gao B-L. Clinical and computed tomographic imaging features of Novel Coronavirus Pneumonia caused by SARS-CoV-2. Journal of Infection 2020. doi: 1016/j.jinf.2020.02.017

19. Xu X, Yu C, Qu J, Zhang L, Jiang S, Huang D, Chen B, Zhang Z, Guan W, Ling Z, Jiang R, Hu T, Ding Y, Lin L, Gan Q, Luo L, Tang X, Liu J. Imaging and clinical features of patients with 2019 novel coronavirus SARS-CoV-2. Eur J Nucl Med Mol Imaging 2020. doi: 10.1007/s00259-020-04735-9

20. China NHCotPsRo. Diagnosis and treatment protocolsof pneumonia caused by a novel coronavirus (trial version

6). http://wwwnhcgovcn/xcs/zhengcwj/202002/8334a8326dd94d329df351d7da8aefc2shtml.

\section{Tables}

Table 1 Demographic of the patients 


\begin{tabular}{|l|l|}
\hline Characteristic & Number of patients (\%) (N=22) \\
\hline Gender & \\
\hline Male & $13(59.1 \%)$ \\
\hline Female & $9(40.9 \%)$ \\
\hline Age & $50 \pm 14(30-79)$ \\
\hline Initial symptoms & \\
\hline Fever & $18(81.8 \%)$ \\
\hline Maximum temperature & \\
\hline$\leq 37.3^{\circ} \mathrm{C}$ & $4(18.2 \%)$ \\
\hline $37.3-38^{\circ} \mathrm{C}$ & $5(22.7 \%)$ \\
\hline $38.1-39^{\circ} \mathrm{C}$ & $9(40.9 \%)$ \\
\hline$\square 39^{\circ} \mathrm{C}$ & $4(18.2 \%)$ \\
\hline Cough & $12(54.5 \%)$ \\
\hline Phlegm & $3(13.6 \%)$ \\
\hline Chest distress or polypnea & $9(40.9 \%)$ \\
\hline Myalgia & $6(27.3 \%)$ \\
\hline Chill or rigor & $5(22.7 \%)$ \\
\hline Fatigue & $16(72.7 \%)$ \\
\hline Diarrhea & $2(9.1 \%)$ \\
\hline Headache or dizziness & $4(18.2 \%)$ \\
\hline dyspnea or chest pain & $4(18.2 \%)$ \\
\hline Loss of appetite & $12(54.5 \%)$ \\
\hline Poor sleep & $4(18.2 \%)$ \\
\hline Laboratory results & \\
\hline Leukocyte count (G/L) & $6.26 \pm 4.46(2.29-19.67)$ \\
\hline$\square 10$ & $2(9.1 \%)$ \\
\hline$\square 4$ & \\
\hline
\end{tabular}

\begin{tabular}{|l|l|}
\hline Neutrophil count (G/L) & $4.62 \pm 4.65(1.05-18.43)$ \\
\hline$\square 6.3$ & $3(13.6 \%)$ \\
\hline$\square 1.8$ & $5(22.7 \%)$ \\
\hline Lymphocyte count (G/L) & $1.12 \pm 0.49(0.47-1.96)$ \\
\hline$\square 1.5$ & $17(77.3 \%)$ \\
\hline C-reactive protein (mg/L) & $30.09 \pm 43.4(3.26-161.66)$ \\
\hline$\geq 10$ & $16(72.7 \%)$ \\
\hline D-dimers (mg/L) & $4.01 \pm 5.99(0.23-20)$ \\
\hline$\geq 0.5$ & $18(81.8 \%)$ \\
\hline Times of negative RT-PCR testing & $3 \pm 1(2-5)$ \\
\hline Numbers of CT scans & $3 \pm 1(1-6)$ \\
\hline $\begin{array}{l}\text { The interval between the adjacent scans } \\
\text { (d) }\end{array}$ & $7 \pm 4(3-20)$ \\
\hline
\end{tabular}


Table 2 CT findings of the patients at initial scan

\begin{tabular}{|l|l|}
\hline Lesions & Number of patients (\%) (N=22) \\
\hline Ground glass opacity (GGO) & $22(100 \%)$ \\
\hline Reticular/interlobular septal thickening & $11(50 \%)$ \\
\hline Consolidation & $14(63.6 \%)$ \\
\hline Distribution pattern of lesions & \\
\hline Peripheral & $15(68,2 \%)$ \\
\hline Random & $1(4.5 \%)$ \\
\hline Diffuse & $6(27.3 \%)$ \\
\hline Number of involved lobes & \\
\hline One lobe & $5(22.7 \%)$ \\
\hline Two lobes & $2(9.1 \%)$ \\
\hline Three lobes & $1(4.5 \%)$ \\
\hline Four lobes & $1(4.5 \%)$ \\
\hline Five lobes & $13(59.1 \%)$ \\
\hline Involved lobes & \\
\hline Right upper lobe & $14(63.6 \%)$ \\
\hline Right middle lobe & $13(59 \%)$ \\
\hline Right lower lobe & $20(90.9 \%)$ \\
\hline Left upper lobe & $16(72.7 \%)$ \\
\hline Left lower lobe & $18(81.8 \%)$ \\
\hline Unilateral & $6(27.3 \%)$ \\
\hline Bilateral & $16(72.7 \%)$ \\
\hline
\end{tabular}

Table 3 CT scores of the involved lobes at initial scan

\begin{tabular}{|l|l|}
\hline Lobe & Score (mean \pm standard deviation) \\
\hline Right upper lobe & $1.41 \pm 1.26$ \\
\hline Right middle lobe & $1.45 \pm 1.37$ \\
\hline Right lower lobe & $2.18 \pm 1.181$ \\
\hline
\end{tabular}

\begin{tabular}{|l|l|}
\hline Left upper lobe & $1.86 \pm 1.32$ \\
\hline Left lower lobe & $2.27 \pm 1.35$ \\
\hline All lobes & $9.18 \pm 5.94$ \\
\hline
\end{tabular}

Table 4 Total CT scores of different groups 


\begin{tabular}{|l|l|}
\hline Group & Score (mean \pm standard deviation) \\
\hline Group 1 ( $\leq 7$ days after symptom onset) & $5.06 \pm 4.22$ \\
\hline Group 2 ( $>7$ to 14 days) & $10.0 \pm 4.491$ \\
\hline Group 3 (>14 to 21 days) & $9.39 \pm 4.792$ \\
\hline Group 4 ( $>21$ days) & $8.70 \pm 3.743$ \\
\hline
\end{tabular}

\section{Figures}
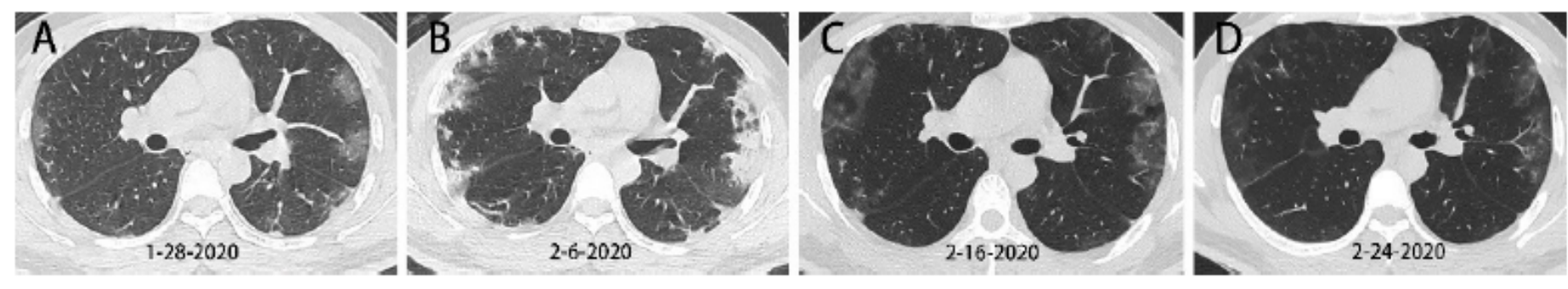

Figure 1

Serial chest CT images of a 32 years old male patient with cough for 5 day. RT-PCR showed negative results for SARS-CoV-2 on January 30, February 4, 2020, respectively. A. Multiple subpleural ground-glass opacity (GGO) affecting bilateral lungs. B. Progression of the disease from multiple GGO to consolidation. $\mathrm{C}$ and $\mathrm{D}$. Absorption of consolidation in bilateral lungs.

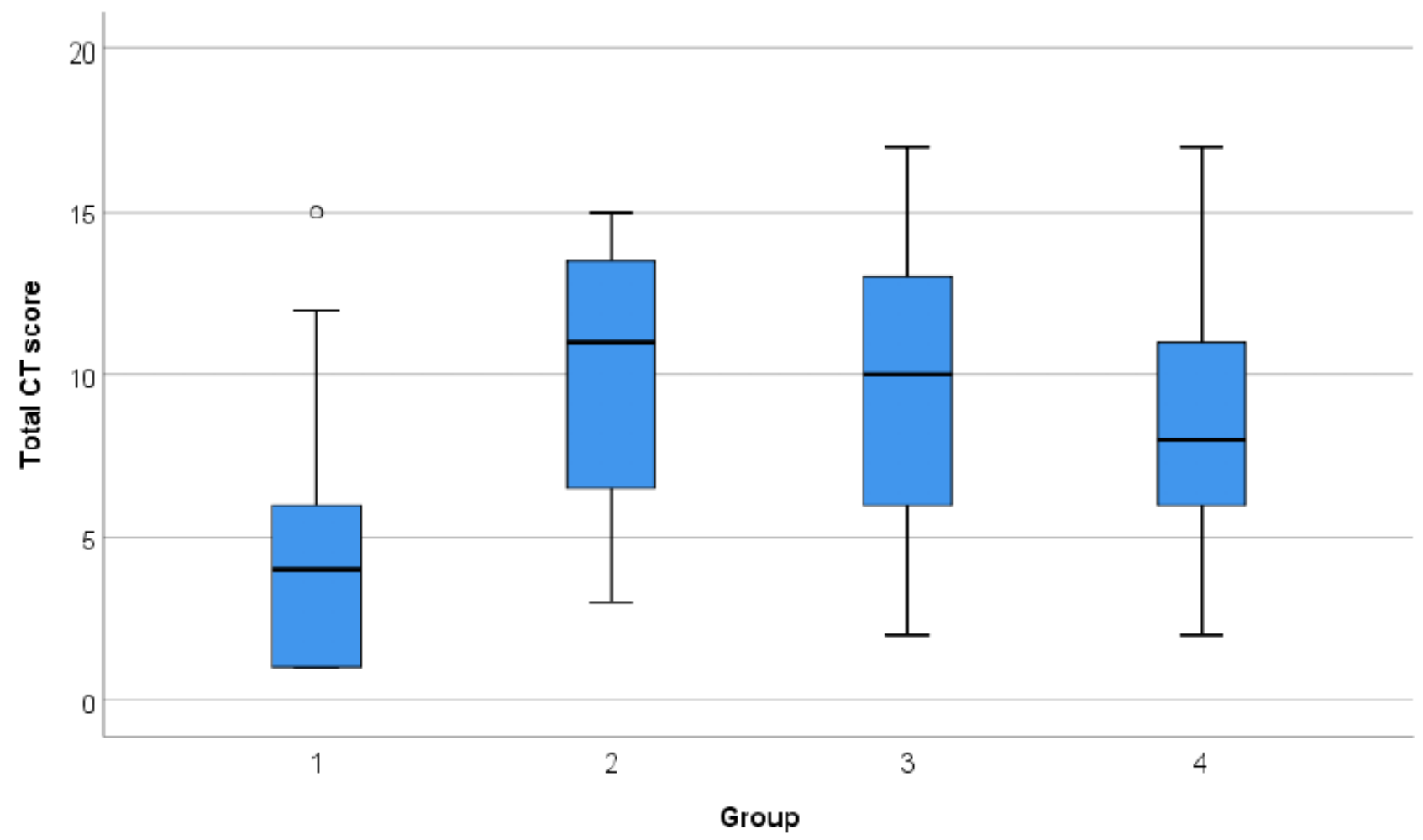

Figure 2 
Total CT score of lung involvement at various timepoints from symptom onset. Bars show the total CT scores on CT scans from patients in group 1 (CT scans performed $\leq 7$ days after symptom onset, $n=18$ ); group 2 (CT scans performed $>8$ to 14 days after symptom onset, $n=11$ ); group 3 (CT scans performed $>14$ to 21 days after symptom onset, $n=18$ ); and group 4 (CT scans performed $>21$ days after symptom onset, $n=23)$.
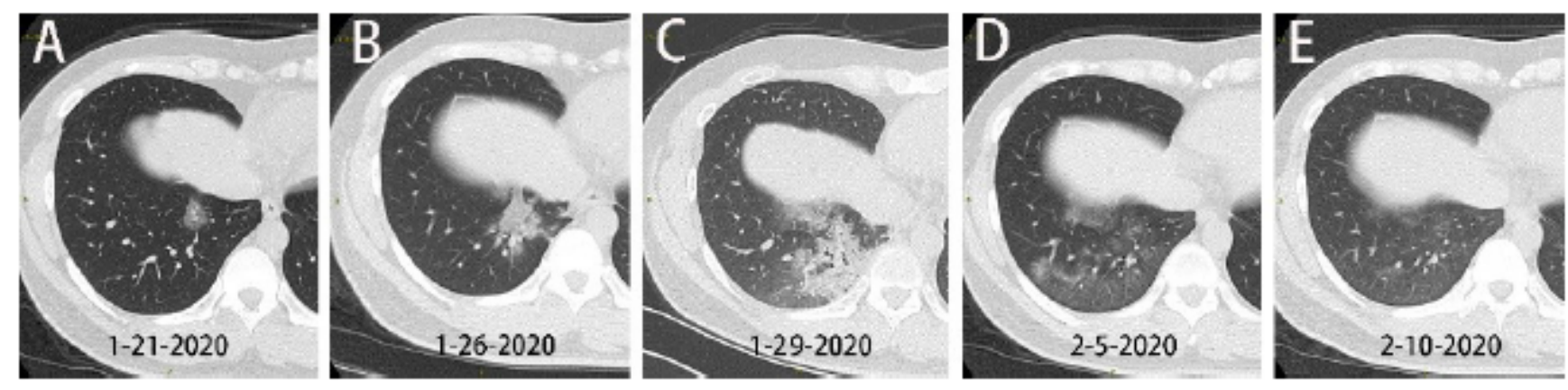

\section{Figure 3}

Serial chest CT images of a 34 years old male patients with fever for 1 day. RT-PCR showed negative results for SARS-CoV-2 on January 28, February 1 and 11, 2020, respectively. A. Focal ground-glass opacity (GGO) affecting the right lower lobe. B and C. Enlarged GGO with consolidation and interlobular septal thickening. D. Absorption of consolidation and interlobular septal thickening with enlarged GGO. E. Absorption of GGO.
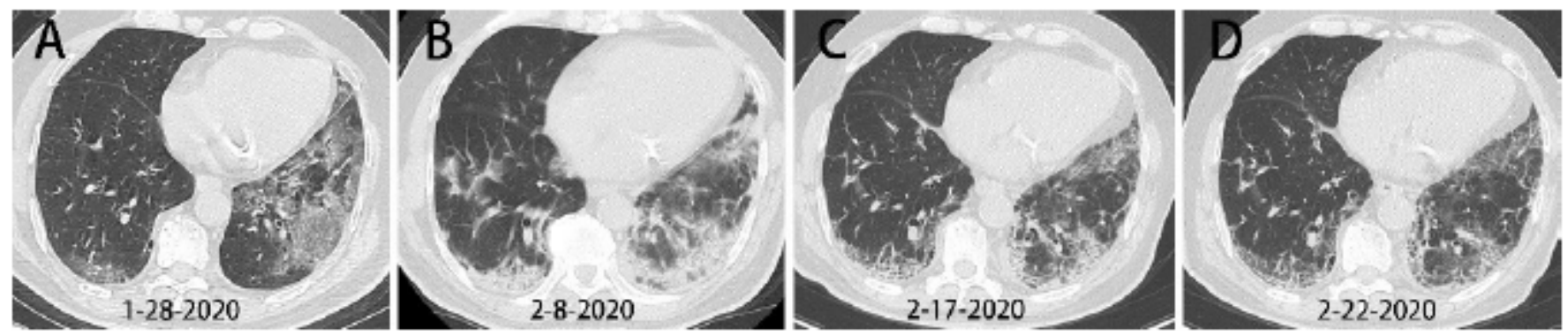

Figure 4

Serial chest CT images of a 69 years old male patient with fever and fatigue for 4 day. RT-PCR showed negative results for SARS-CoV-2 on January 30, February 2, 2020, respectively. A. Subpleural and diffuse ground-glass opacity (GGO) with interlobular septal thickening affecting the right lower lobe and left lower lobe, respectively. B. Increased consolidation with interlobular septal thickening in bilateral lungs. C. Partial absorption of consolidation with remaining interlobular septal thickening and reticular opacity. D. Continued absorption of consolidation in bilateral lungs.

\section{Supplementary Files}


This is a list of supplementary files associated with this preprint. Click to download.

- supplementalmaterial.pdf 